\title{
Attribute Analysis of Aridity Variability in North Xinjiang, China
}

\author{
Yanfeng Wu, ${ }^{1,2}$ Guangxin Zhang, ${ }^{1}$ Hong Shen, ${ }^{1}$ Y. Jun Xu, ${ }^{3}$ and Batur Bake ${ }^{2}$ \\ ${ }^{1}$ Key Laboratory of Wetland Ecology and Environment, Northeast Institute of Geography and Agroecology, \\ Chinese Academy of Sciences, 4888 Shengbei Street, Changchun 130102, China \\ ${ }^{2}$ College of Grassland and Environmental Science, Xinjiang Agricultural University, 311 East Nongda Road, Urumqi 830052, China \\ ${ }^{3}$ School of Renewable Natural Resources, Louisiana State University Agricultural Center, 227 Highland Road, \\ Baton Rouge, LA 70803, USA \\ Correspondence should be addressed to Guangxin Zhang; zhgx@iga.ac.cn
}

Received 9 January 2016; Revised 25 April 2016; Accepted 4 May 2016

Academic Editor: Hisayuki Kubota

Copyright (c) 2016 Yanfeng Wu et al. This is an open access article distributed under the Creative Commons Attribution License, which permits unrestricted use, distribution, and reproduction in any medium, provided the original work is properly cited.

Identifying the dominant meteorological factors affecting aridity variability can improve our understanding of climate change and its future trend in arid and semiarid regions. This study investigated the spatiotemporal aridity variability in North Xinjiang, China, from 1961 to 2013, based on the UNESCO aridity index (precipitation/potential evapotranspiration), and analyzed its association with meteorological factors. The results suggest that North Xinjiang is becoming more humid with an increasing trend in aridity index. Precipitation, temperature, and relative humidity have positive correlation with aridity, and evapotranspiration, sunshine hours, and wind speed have negative correlation with aridity. Wind speed and sunshine hours have a higher sensitivity and more contribution to aridity. This study provides an understanding of the effect of recent climate change on drought in northwest China.

\section{Introduction}

Climate change can alter the frequency, intensity, and duration of precipitation in many regions in the world. Some regions may become drier and while other regions may become wetter [1-4]. North Xinjiang (NX) of China is one of the arid and semiarid regions in the world that are highly vulnerable to climate change. The region is within the great Eurasian steppe land and its vast steppe ecosystem of temperate grasslands, savannas, and shrub lands is sensitive to change in water availability $[5,6]$. Knowledge of the spatial and temporal variation of dry-wet conditions and its longterm relationship with other climatic factors in North Xinjiang can help develop adaptive management strategies and plans to ensure the continued conservation and sustainability of agricultural development in the region [7-9].

Aridity, as defined by the shortage of moisture, is essentially a climatic phenomenon that is based on average climatic conditions over a region, representing potential water availability at various scales, especially at large scales [4, 10]. For the regions, aridity indices are commonly used to detect potential risks of occurrence and severity of aridity changes and spatiotemporal patterns. To this end, many aridity indexes, that is, Budyko's aridity index [11], aridity intensity index [12], Pinna aridity indices [13], De Martonne aridity index [14, 15], Thornthwaite aridity index [16, 17], and UNESCO aridity index [18], have also been proposed and used based on different variables and parameters. One of these aridity indices, the UNESCO aridity index (AI), defined by the ratio of precipitation $(P)$ to annual potential evapotranspiration $\left(\mathrm{ET}_{0}\right)$, has been widely used $[4,19,20]$. The UNESCO aridity index realistically presents the water as it considers not only the total precipitation as water inflows but also the water loss through evaporation. Therefore, the UNESCO AI is used in this study as a quantitative indicator to describe the water deficiency/aridity associated with the NX.

Many studies, conducted on climate variability in dryness and wetness in western China, indicated that warm-wet trend occurred in northwest China [21-24]. However, no consensus has been reached for factors affecting aridity in Xinjiang Province. Depending on potential evapotranspiration and 


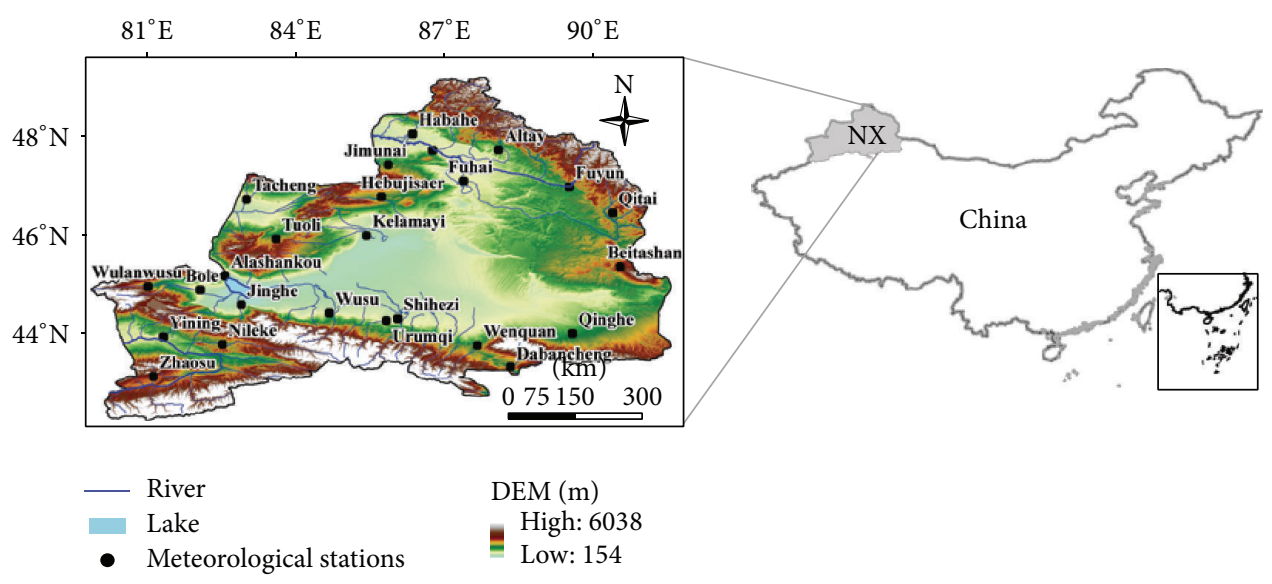

FIgURE 1: Geographical location of North Xinjiang, China.

precipitation data produced by HadCM3 under IPCC A2 and B2 scenarios, Li and Zhou [25] studied the trends in dryness in Xinjiang between 1961 and 2099. However, these studies only focused on the changing dryness and wetness; they did not further explore the driving factors behind such a trend. Apart from the total amount of precipitation, meteorological factors, such as temperature, wind speed, relative humidity, and shine hours, could be the potential drivers affecting the $\mathrm{ET}_{0}$ and hence the dryness under the context of climate change $[26,27]$. Therefore, the variability of these meteorological factors and their contribution to aridity deserve a close look.

This study aimed to analyze the spatiotemporal variation of the aridity in the NX over the past five decades. Specifically, the objectives of the study were to (1) analyze the spatial and temporal variations of aridity index and (2) determine the relations and sensitivity of aridity index to meteorological factors.

\section{Materials and Methods}

2.1. Study Area. North Xinjiang $\left(75-95^{\circ} \mathrm{E}, 42-49^{\circ} \mathrm{N}\right)$ is located in northwest China, the hinterland of Eurasia (Figure 1). The region is dominated by temperate continental arid climate, characterized by abundant sunshine, large variations in temperature fluctuation, extremely low precipitation, and high evaporation rates. Long-term monthly average temperatures range between -4 and $9^{\circ} \mathrm{C}$, with the hottest month in August, and the coldest month in January. On average, annual precipitation is between 150 and $200 \mathrm{~mm}$, with 140 to 185 days each year free of frost [28-30]. The climate of NX is representative of arid regions in China and is also similar to those in other arid regions in the world. Ecosystems in the region are rather fragile for the arid climate change features $[31,32]$. Under the background of global climate warming, the climate in Xinjiang is transforming from warm-dry to warmwet trends [23].

2.2. Calculation and Spatial Interpolation of Aridity Index. This study used daily weather records during the period
1961-2013 collected from 25 meteorological stations in NX. The data were provided by the Chinese Meteorological Data Sharing Service System (CMDSSS; http://data.cma.cn/) and weather parameters including precipitation $(P)$, mean temperature $(T)$, maximum temperature $\left(T_{\max }\right)$, minimum temperature $\left(T_{\min }\right)$, sunshine hours $(\mathrm{SH})$, wind speed (WS), and relative humidity $(\mathrm{RH})$ were selected for this study. The data quality for these weather records has been controlled by China Meteorological Administration and had been proven to be unmistakable by means of the extreme value examination and time synchronization detection. Few missing stations have missing data which were filled by using linear regression method. The aridity indexes (AI) as defined by UNESCO (1979) were calculated based on precipitation and potential evapotranspiration (i.e., $\mathrm{AI}=P / \mathrm{ET}_{0}$ ) for 25 meteorological stations in NX. The aridity index increases as conditions become more humid. Potential evapotranspiration was calculated using the Penman-Monteith method (for $\mathrm{ET}_{0}[33]$ ). The anomaly of aridity was obtained by minus the average value of aridity for each meteorological station form over 25 stations from 1961 to 2013. The results were spatially interpolated with the Kriging method in ArcGIS for the entire North Xinjiang.

2.3. Sensitive Analysis. Sensitive analysis mathematically examined the relative sensitivity coefficient between dependent and independent variables. It has been widely used to quantify the contribution of meteorological factors to evapotranspiration [34-36]. Previous study is also adopted to confirm AI sensitivity to climatic factors using [37]. Here, we used it to investigate the driving factors to aridity in NX. Relative sensitivity coefficient $(S)$ was calculated based on McCuen [38]:

$$
S=\lim \left(\frac{\Delta \mathrm{AI} / \mathrm{AI}}{\Delta V / V}\right)=\frac{\partial \mathrm{AI}}{\partial V} \cdot \frac{|V|}{\mathrm{AI}} .
$$

A first-order Taylor series approximation was applied to calculate $S[36]$ :

$$
S=\frac{\Delta \mathrm{AI}}{\Delta V} \cdot \frac{|V|}{\mathrm{AI}}
$$


where $\Delta V$ is the relative change (RC) of model input value $V$ (involving six variables here: temperature, precipitation, wind speed, relative humidity, sunshine hours, and $\mathrm{ET}_{0}$ ) and $\Delta \mathrm{AI}$ is the relative change of AI induced by $\Delta V$. Relative change was indicated by the percentage of change in the past 53 years to an absolute average value $(|\operatorname{av}|)$ :

$$
\mathrm{RC}=\frac{53 \cdot V}{|\mathrm{av}|} .
$$

Coefficient $S$ refers to changes in AI associated with the change in the meteorological variable $V$. If $S$ is 0.5 , it means that $10 \%$ of change in $V$ would cause $5 \%$ of change in AI if the other meteorological variables remain constant. The higher the coefficient $S$ is, the larger the impact the meteorological variable imposes on $\mathrm{AI}$ is. The positive (negative) value of $S$ means that AI will change in accordance (disaccord) with $V_{i}$. $S$ is dimensionless which makes it convenient to sort the order by meteorological influence. In order to assess to which level the sensitivity of meteorological factor to AI would reach, $S$ was ranked into four classes (Table 1).

2.4. Contributions Analysis. In multiple regression model, the relative contribution of an independent variable can be defined as follows [39]:

$$
\begin{aligned}
& Y_{s}=a X_{1 s}+b X_{2 s}+c X_{3 s}+\cdots, \\
& \eta_{i}=\frac{|a|}{|a|+|b|+|c|+\cdots},
\end{aligned}
$$

where $Y_{s}$ is the dependent variable (AI), $X_{1 s}, X_{2 s}, X_{3 s}, \ldots$ are the independent variables (precipitation, temperature, sunshine hours, etc.), $a, b, c, \ldots$ is the regression coefficient, and $\eta_{i}$ is the relative contribution related to the independent variable.

2.5. Cross-Wavelet Analysis. In this study, we applied wavelet analysis, which has been broadly used in meteorological, hydrological, and environmental studies to assess temporal characteristics of a time series $[40,41]$. We estimate the multiscale phase relationship between the components of the two time series (the aridity and meteorological factors) using wavelet coherences (WTCs) [42, 43]. WTCs show localized correlation coefficients between aridity and meteorological factors in time-frequency space and, thus, can expose the area with significant coherence between two Continuous Wavelet Transformations (CWTs) even though the common power is low [44]. With the approach of Torrence and Webster [45], we define the WTCs as

$$
R^{2}(s, t)=\frac{\left|S\left(s^{-1} W_{X Y}(s, t)\right)\right|^{2}}{S\left(s^{-1}\left|W_{X}(s, t)\right|^{2}\right) \cdot S\left(s^{-1}\left|W_{Y}(s, t)\right|^{2}\right)},
$$

where $S$ is a smoothing operator. The scales in time and frequency over which $S$ is smoothing define the scales at which the coherence measures the covariance. The difference between the phase angles determined by the cross-wavelet and coherence methods is essentially only the smoothing
TABLE 1: Sensitivity levels in the time they are cited.

\begin{tabular}{lc}
\hline Sensitivity coefficient & Sensitivity levels \\
\hline $0.00 \leq|S|<0.05$ & Small to negligible \\
$0.05 \leq|S|<0.20$ & Medium \\
$0.20 \leq|S|<1.00$ & High \\
$|S| \geq 1$ & Very high \\
\hline
\end{tabular}

used in the coherence method. Therefore, for simplicity, we only quote the phase angles and the confidence intervals determined for the coherence analysis; see Jevrejeva et al. [42] for an extensive discussion. We used the circular mean of the phase for those regions with higher than 5\% statistical significance and those which are outside the cone of influence (COI) to quantify the phase relationship. We calculate the 95\% confidence angle of the mean phase assuming a Von Mises distribution. The WTCs were calculated using MATLAB code provided by Grinsted et al. [44].

\section{Results}

3.1. Spatial and Temporal Aridity. The spatial distributions of the average annual aridity exhibited an increasing trend from northwest to southeast over the study region in NX (Figure 2(a)). The anomaly of aridity exceeding the average annual aridity was centralized in northern NX, while southern NX is dominated by relatively low values. Among the 25 stations, the ones distributed in the northeastern and northern NX showed stronger increasing trend in aridity than the southern part (see Figure 2(b); statistically significant at $99 \%$ confidence level). The uneven distribution of aridity anomaly is mainly caused by regional climate characteristics created by topography heterogeneity [36]. In addition, the uneven influence of climate change on regional meteorological factors may have great influence on the strength of aridity change [46].

The interannual temporal change in the aridity anomaly in NX is plotted in Figure 3, which presents an overall increasing trend during 1961-2013. The lowest value occurred in $1960 \mathrm{~s}$, with the minimum anomaly value of -0.09 seen in 1961; the highest happened in 2000s, soaring up to 0.10 in 2010. A turning point appeared in the late 1980s and the early 1990s, before which the aridity was apparently lower than the average. Linear regression revealed that the aridity of NX increased at the rate of $0.071 /$ decade over the past 53 years. It also suggests that the NX has transformed from a relative dry period to a wet period by increase in $P$ and $T$ (Figures 4(a) and 4(c)). However, $\mathrm{ET}_{0}, \mathrm{RH}, \mathrm{SH}$, and WS experienced a downward trend at different levels (Figures 4(b), 4(d), 4(e), and 4(f)).

3.2. Relations and Sensitivity of Aridity with Meteorological Factors. To further identify the relationship between each meteorological factor and AI, linear correlation and sensitivity analysis were conducted. Figure 5 maps the point-scaled correlation between the annual time series of AI and four meteorological factors ( $P$ and $\mathrm{ET}_{0}$ are excluded due to the fact that they are the input variables when computing AI) 

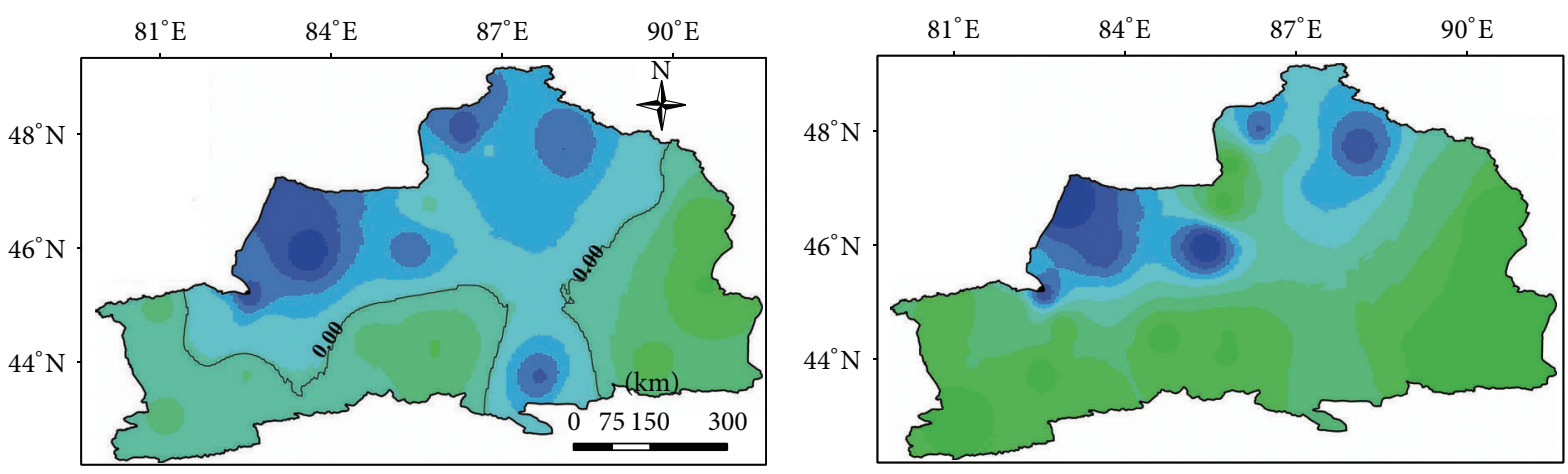

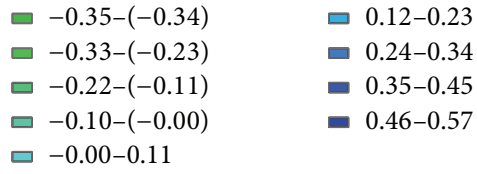

(a)

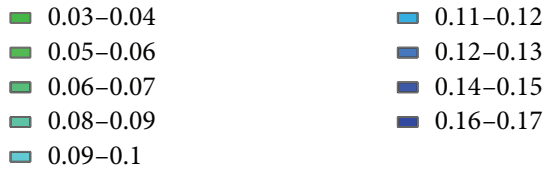

(b)

Figure 2: Spatial distributions of anomalies (a) and trends (b) of AI in North Xinjiang, China, between 1961 and 2013.

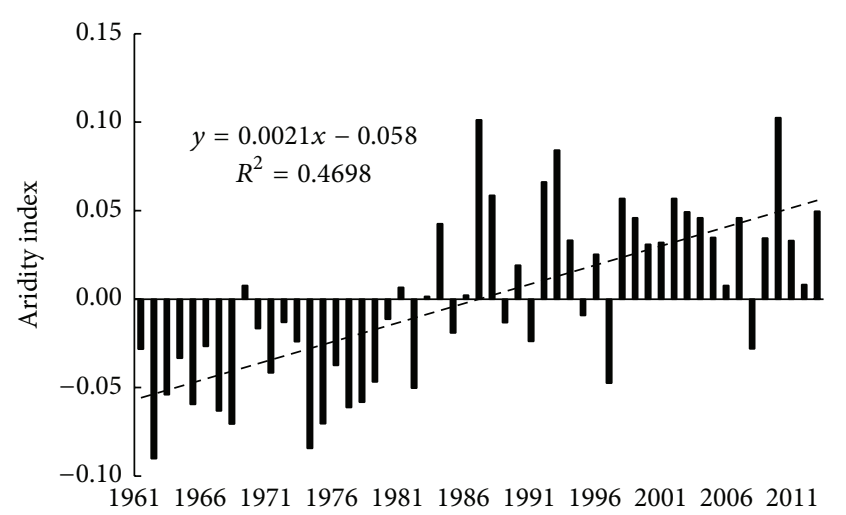

(Year)

Figure 3: Change in the AI anomaly in North Xinjiang, China, during 1961-2013.

in the NX. As shown in Figure 5(a), $T$ mainly had positive correlation with the aridity index in most part of NX except for the northern tip (five stations had negative correlation coefficients). RH increased with $\mathrm{AI}$ at all stations, with 11 reaching the confidence level of $99 \%$ and three for $95 \%$ (Figure 5(b)). SH and WS had negative correlation with AI, which can be obviously reflected by the significant confidence level of about $40 \%$ of total stations in NX (Figures 5(c) and $5(\mathrm{~d}))$.

Regional sensitivity of the aridity to different meteorological factors was demonstrated in Figures 6 and 7, from which the potential main contributors to the aridity trends in NX can be detected. The sensitivity of $P$ and $T$ to aridity was positive over the whole NX, while the sensitivity of $\mathrm{ET}_{0}$, $\mathrm{RH}, \mathrm{SH}$, and WS to aridity had more stations which were negative than stations which were positive in NX. According to the sensitivity category in Table 1 , AI was not sensitive to $P\left(0.0005 \sim 0.0016\right.$; see Figure 6(a)) and $\mathrm{ET}_{0}(0.0003 \sim 0.0519$; see Figure 6(b)) but has medium and high sensitivity to $T$ (0.022 0.232; see Figure 6(c)) and RH (0.011 1.964; see Figure $6(\mathrm{~d}))$; it tends to be very sensitive to $\mathrm{SH}(0.005 \sim$ 2.259; see Figure 6(e)) and WS (0.014 2.093; see Figure 6(f)). Therefore, the sensitivity of meteorological factors to aridity is following the following order: high sensitivity of $\mathrm{SH}$ and WS, medium sensitivity of $T$ and $\mathrm{RH}$, low sensitivity of $P$ and $\mathrm{ET}_{0}$.

The relative contribution of meteorological factors to the aridity changes is shown in Figure 8. WS was the biggest contributor in most stations (contribution rate: $37.48 \%$ ), followed by SH with an average rate of $29.94 \%$. The contribution from $P$ only takes up $12.26 \% \mathrm{~T}$; $\mathrm{RH}$ and $\mathrm{ET}_{0}$ had the least contribution to AI variation, with the rate ranging between 1.7 and 5.6.

Via Figure 9, we found that $\mathrm{ET}_{0}, T, \mathrm{RH}, \mathrm{SH}$, and WS presented a consistently significant coherence $(P<0.05)$ with annual aridity at different period range band throughout the study period: mainly significant in 4-5 years and 1417 years for $\mathrm{ET}_{0} ; 2-4$ years during 1980 s and 6-8 years for T; 3-8 years for RH; 4-8 years for $\mathrm{SH}$; 4-7 years for WS during 1970s. $\mathrm{ET}_{0}, \mathrm{~T}, \mathrm{RH}, \mathrm{SH}$, and WS showed the regions of power significant at the $95 \%$ level, but $P$ was not. $P$ showed a consistency in phase relationship (i.e., left-pointed arrows) with annual aridity from 1961 to 2013 but not reaching the confidence level of $95 \%$. The significant relationships between annual aridity and $\mathrm{ET}_{0}$ were generally antiphase, as with the results for annual aridity and $P, T, \mathrm{RH}, \mathrm{SH}$, and $\mathrm{WS}$, while the relationship between annual aridity and $\mathrm{RH}$ was antiphase.

\section{Discussion}

This study showed that the climate in North Xinjiang, China, is becoming wetter in the past half century. This is in accordance with the finding by Shi et al. [23], which suggested a transformation from a warm-dry to a warm-humid climate 


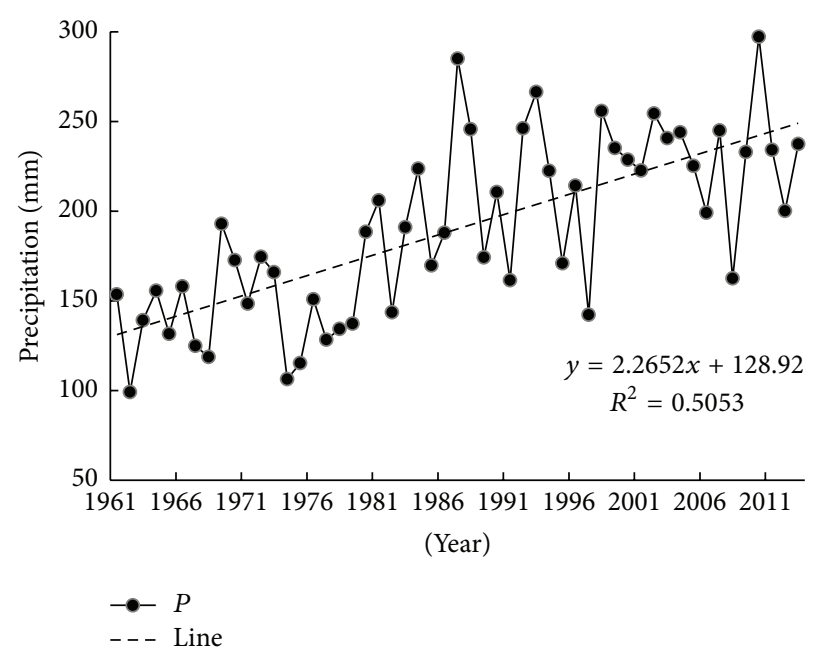

(a)

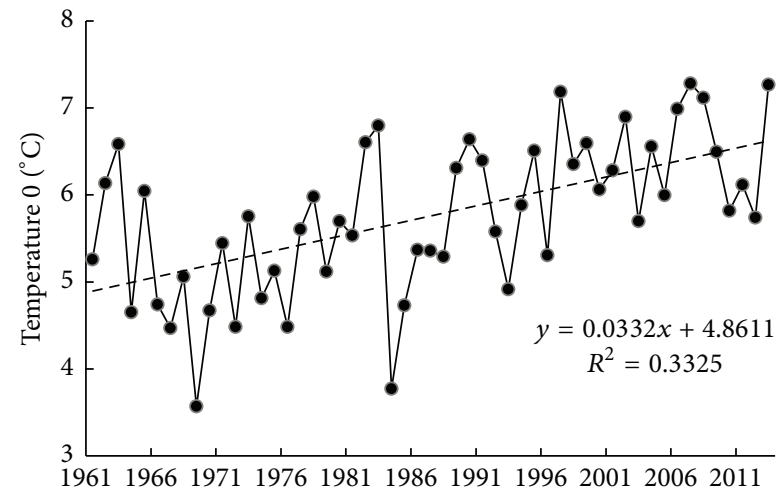

(Year)

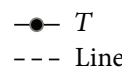

(c)

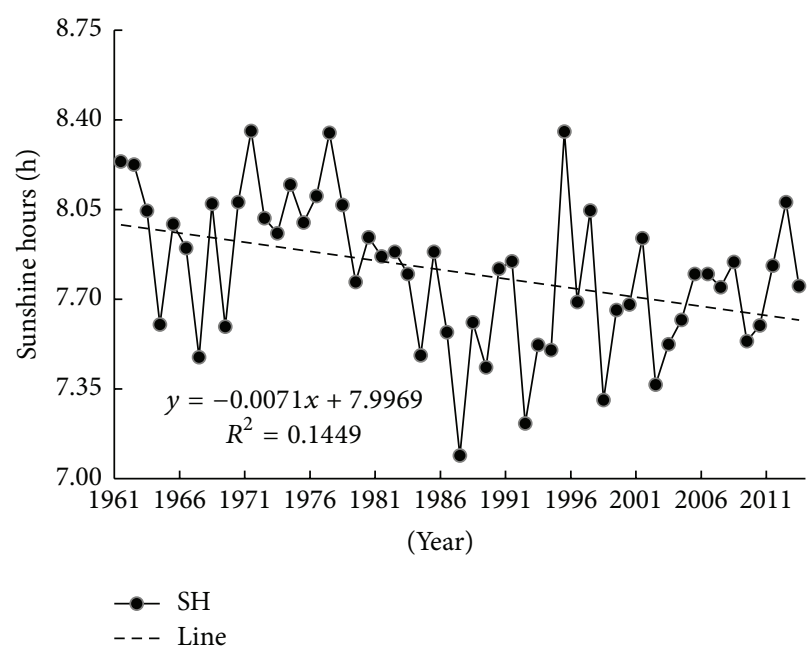

(e)

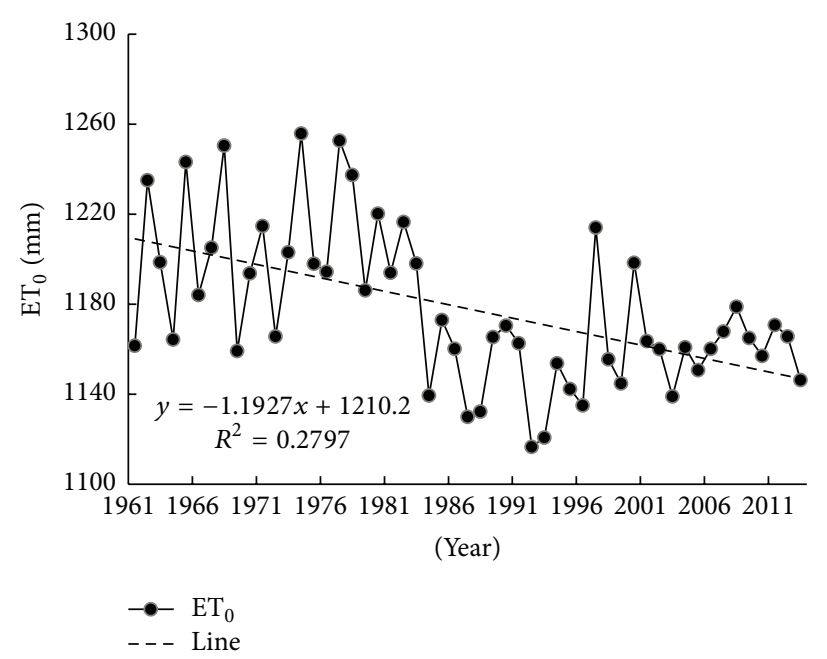

(b)

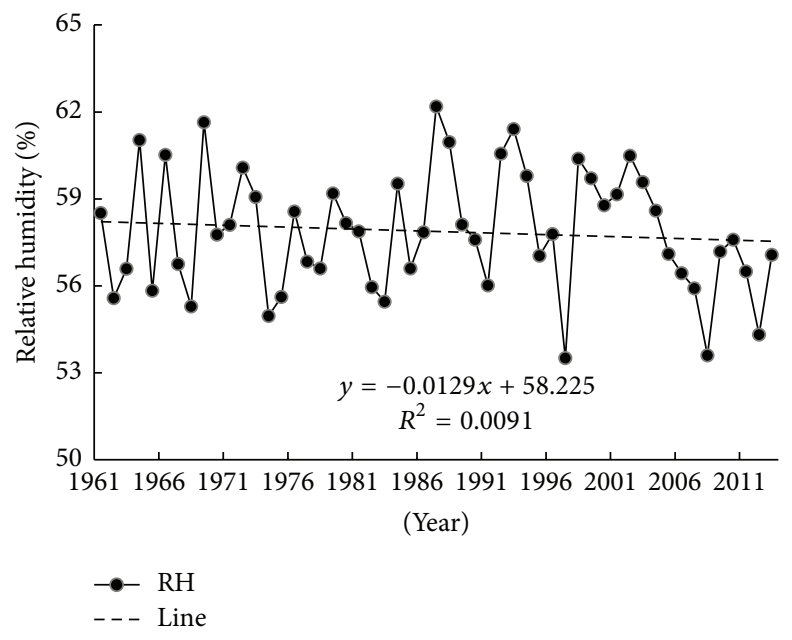

(d)

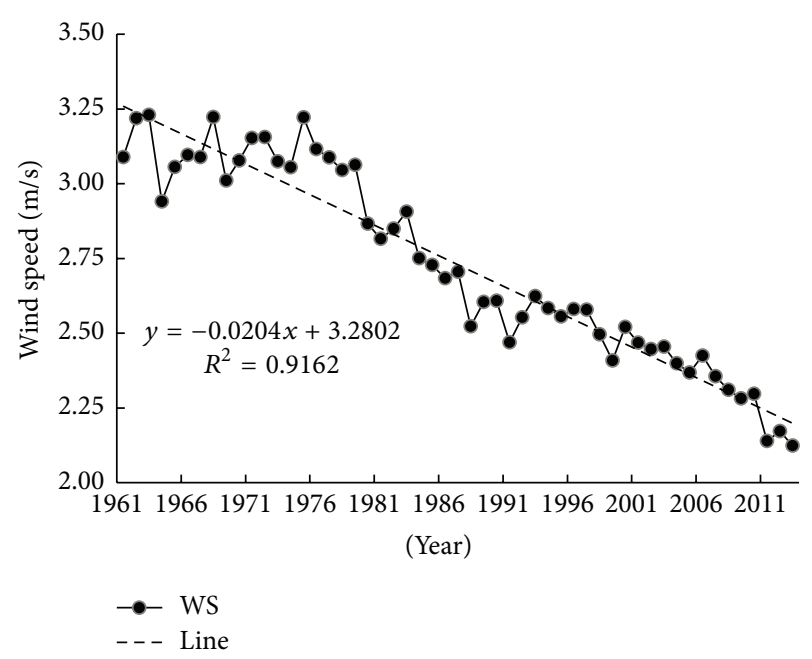

(f)

Figure 4: Time series and its trend associated with the six meteorological factors over North Xinjiang, China, during 1961-2013. 


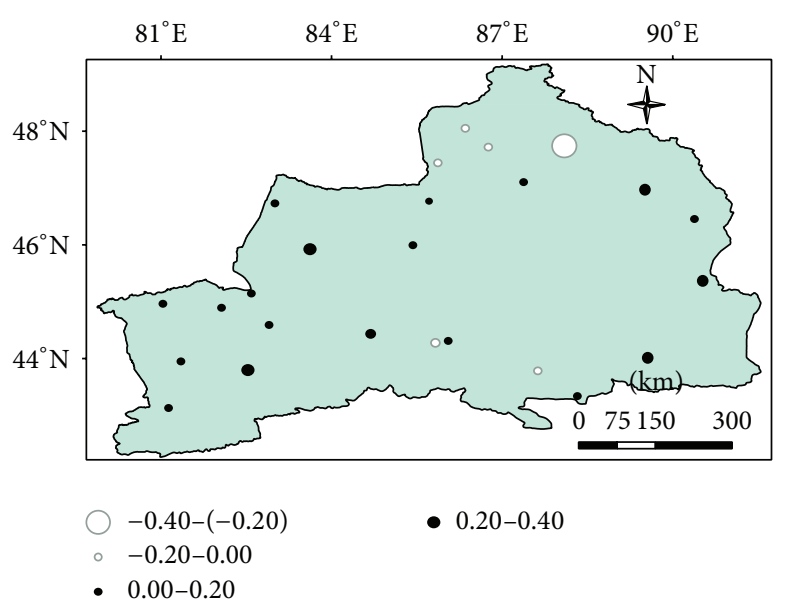

(a) $T$

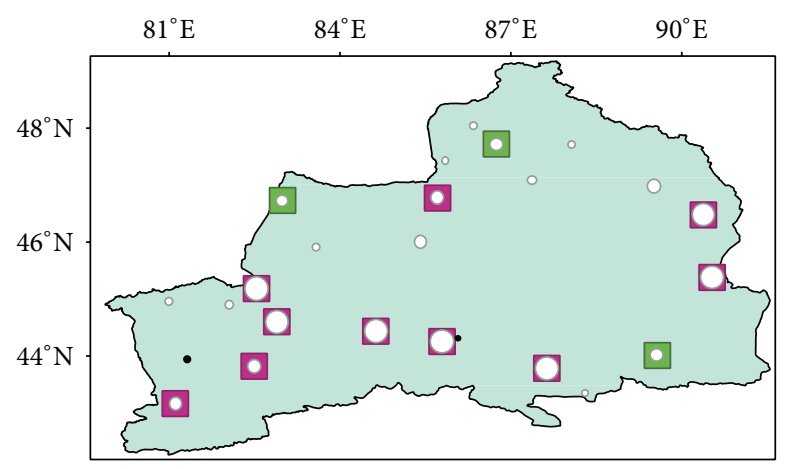

Confidence level of $95 \%$ Confidence level of $99 \%$ $-0.60-(-0.40)$

(c) $\mathrm{SH}$
- $-0.20-(-0.00)$

- $0.00-0.20$
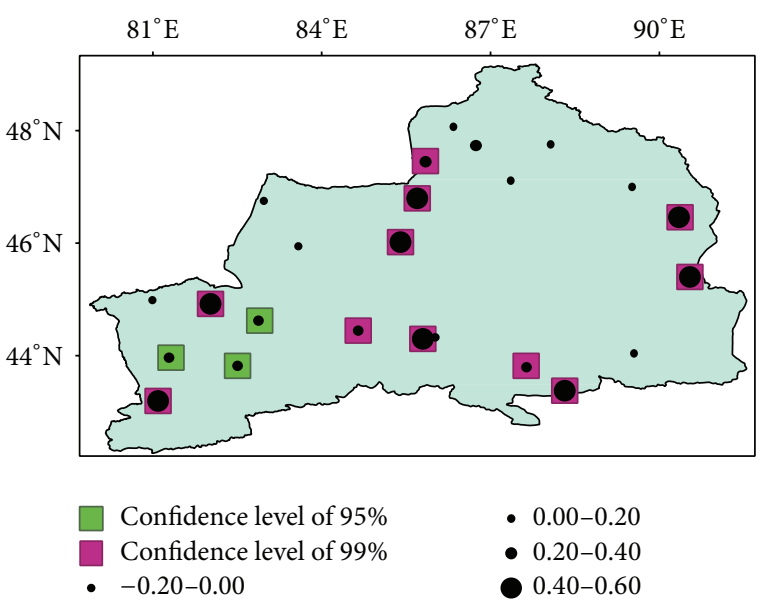

(b) $\mathrm{RH}$

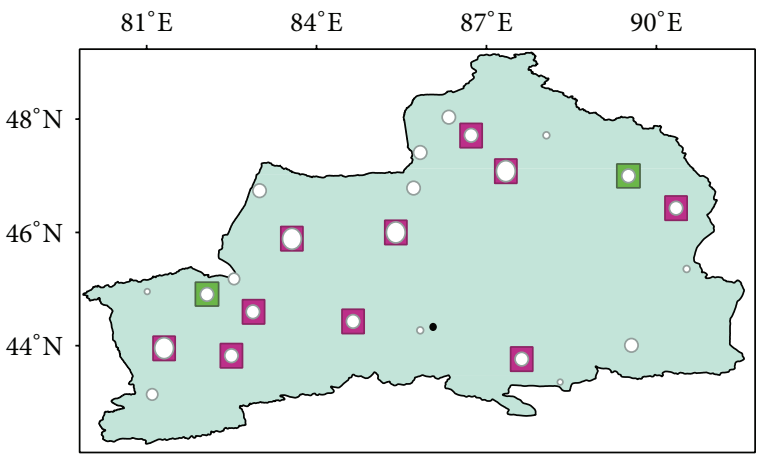

Confidence level of $95 \%$

Confidence level of $99 \%$

-0.60-(-0.40)

(d) WS

FIGURE 5: Spatial distribution of temporal correlations of time series AI with temperature (T), relative humidity (RH), wind speed (WS), and sunshine hours (SH) in North Xinjiang, China.

in northwest China. It is worth noting that the wetting trend in NX may be stronger than that in northwest China and many other regions in the world [4, 23, 47]. Wind speed and sunshine hours are highly sensitivity to AI and thus become the key contributor to the change of AI in NX. Previous researchers also indicate that wind speed and sunshine tend to be the primary cause for the decreased in $\mathrm{ET}_{0}$ in most climate regions in China, especially in its northwestern parts $[48,49]$. Declining trend found in mean surface wind speed in NX was mainly caused by the weakening of the Siberian high pressure and meridional circulation, the decreasing of land-sea temperature difference and pneumatic pressure difference, and the strengthening of zonal circulation influenced by global climate change $[27,50]$.

Asadi Zarch et al. [4] and Feng and Fu [47] suggested that precipitation plays a key role in characterizing the aridity variations in hyperarid areas. However, close inspection in this paper indicated that the precipitation has a lower sensitivity and contribution to aridity in NX. It is because the total precipitation is rare and cannot efficiently meet the robust evapotranspiration in NX $[7,28]$. Therefore, the main driving factors of the increasing trend of aridity index in NX are controlled by the decreasing wind speed and increasing sunshine hours.

$\mathrm{ET}_{0}$ and $P$, the two derivatives of the UNESCO aridity index, as well as the key impact factors for arid and semiarid ecosystem and agricultural production, presented substantially decreasing and increasing trend in NX, respectively. Thus, the trends in $P$ and $\mathrm{ET}_{0}$ increase aridity index (becoming wetter), which means the drought severity would potentially be alleviated and the need for agricultural irrigation would be reduced [19]. In addition, with a significant increase in precipitation in winter, the amount of snowmelt in mountain areas will increase continually, which will supply more water for local farming system in oasis [21, 25, 26]. Obviously, the difference between water surplus and deficit revealed by significant decreasing trend of $\mathrm{ET}_{0}$ and increasing trend of precipitation indicated that the output water by evapotranspiration of the region gradually declines. Therefore, it is definite that the wetting trend becomes stronger. Although the region is becoming wetter, the drought severity has not been fundamentally changed and the gap between water 


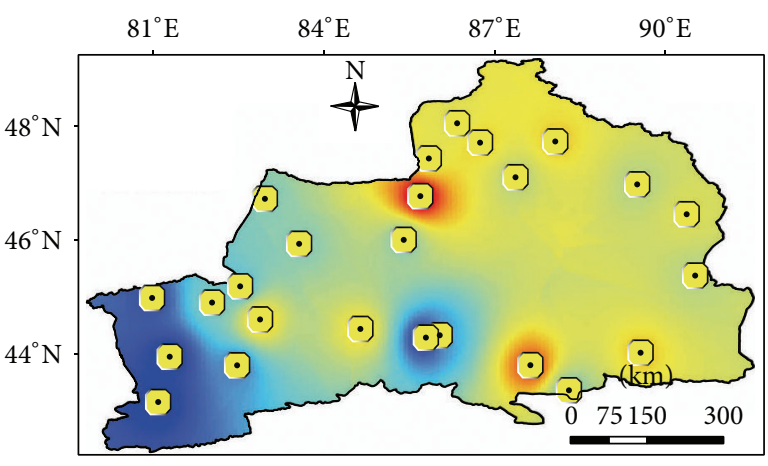

- Small to negligible

D Positive

High: 0.0005

Low: 0.0016

(a) $P$

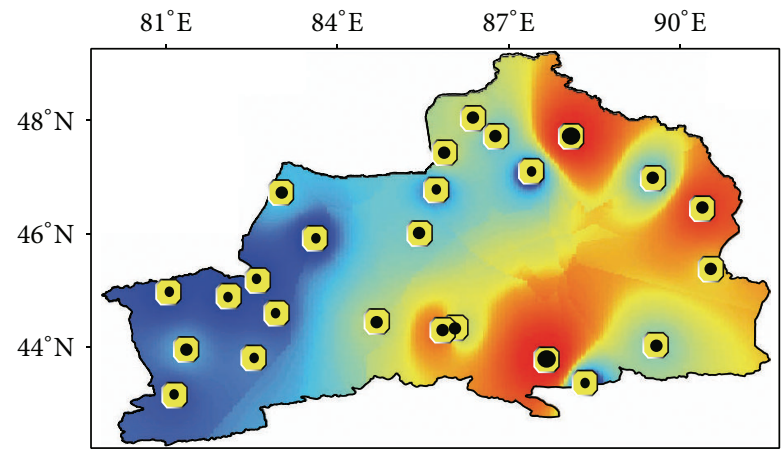

- Small to negligible

- Medium

- High

Dositive

High: 0.2319

Low: 0.0220

(c) $T$

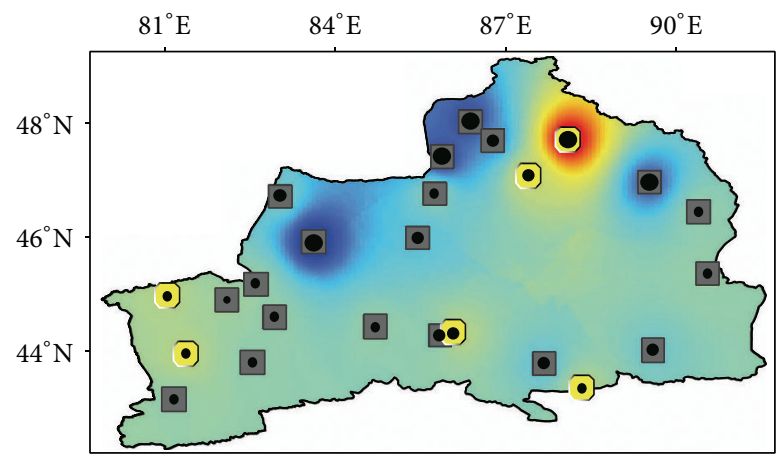

- Small to negligible

- Medium

- High

- Very high

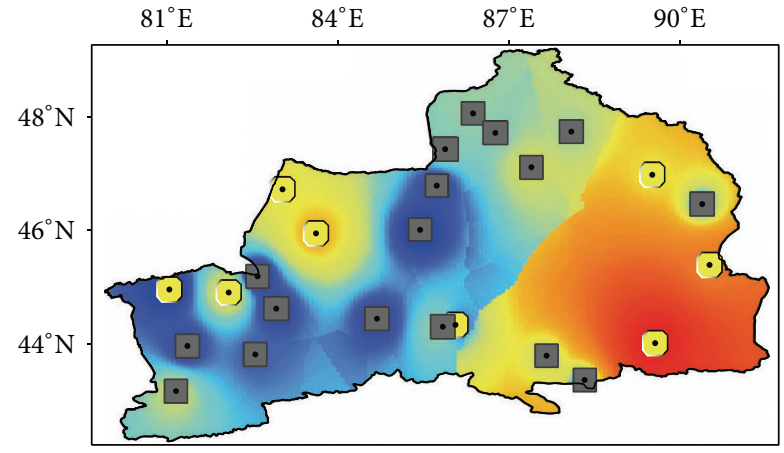

- Small to negligible

Negative

] Positive

High. 0.051 b) $\mathrm{ET}_{0}$

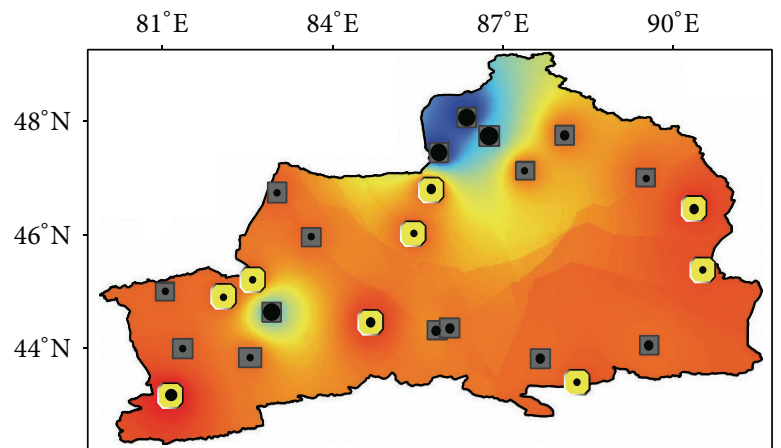

- Small to negligible

Positive

- Medium

- High

- Very high

- Negative

- High: 0.2029

Low: -1.9637

(d) $\mathrm{RH}$

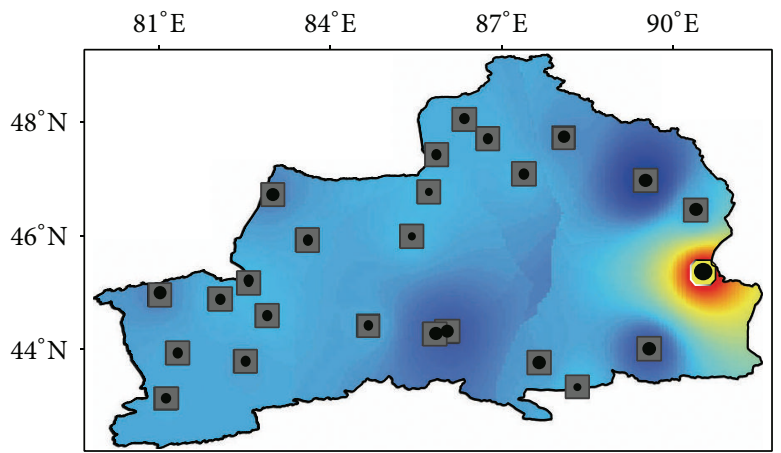

- Small to negligible

- Medium

Positive

- High

- Very high
Negative

- High: 2.0909

Low: -0.8619

(f) WS

(e) $\mathrm{SH}$ 


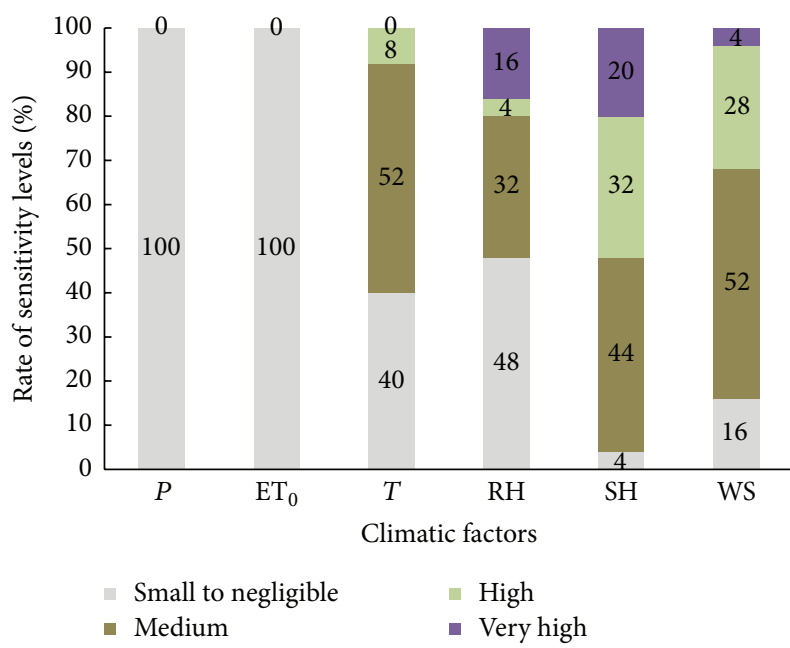

(a)

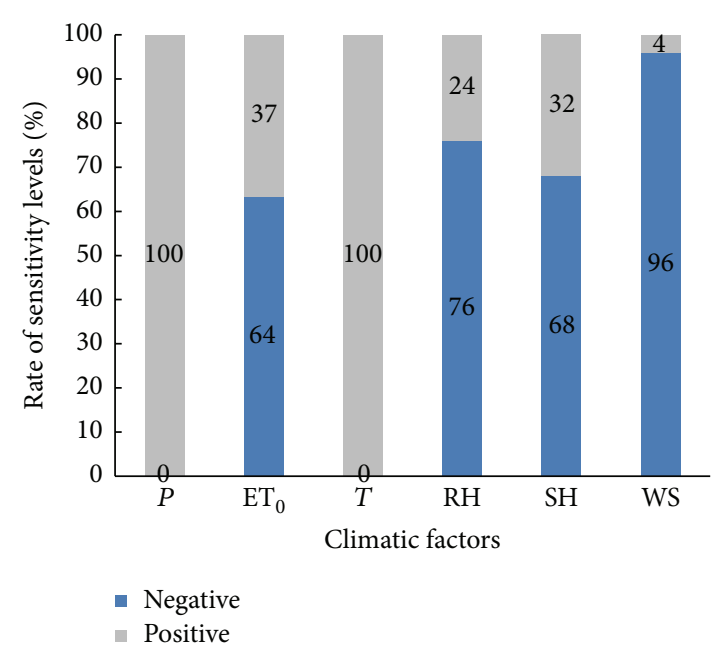

(b)

FIGURE 7: Percentage of the total testing stations distributed over different sensitivity levels (a) and over positive and negative sensitive coefficients (b) among the six meteorological factors in North Xinjiang, China.

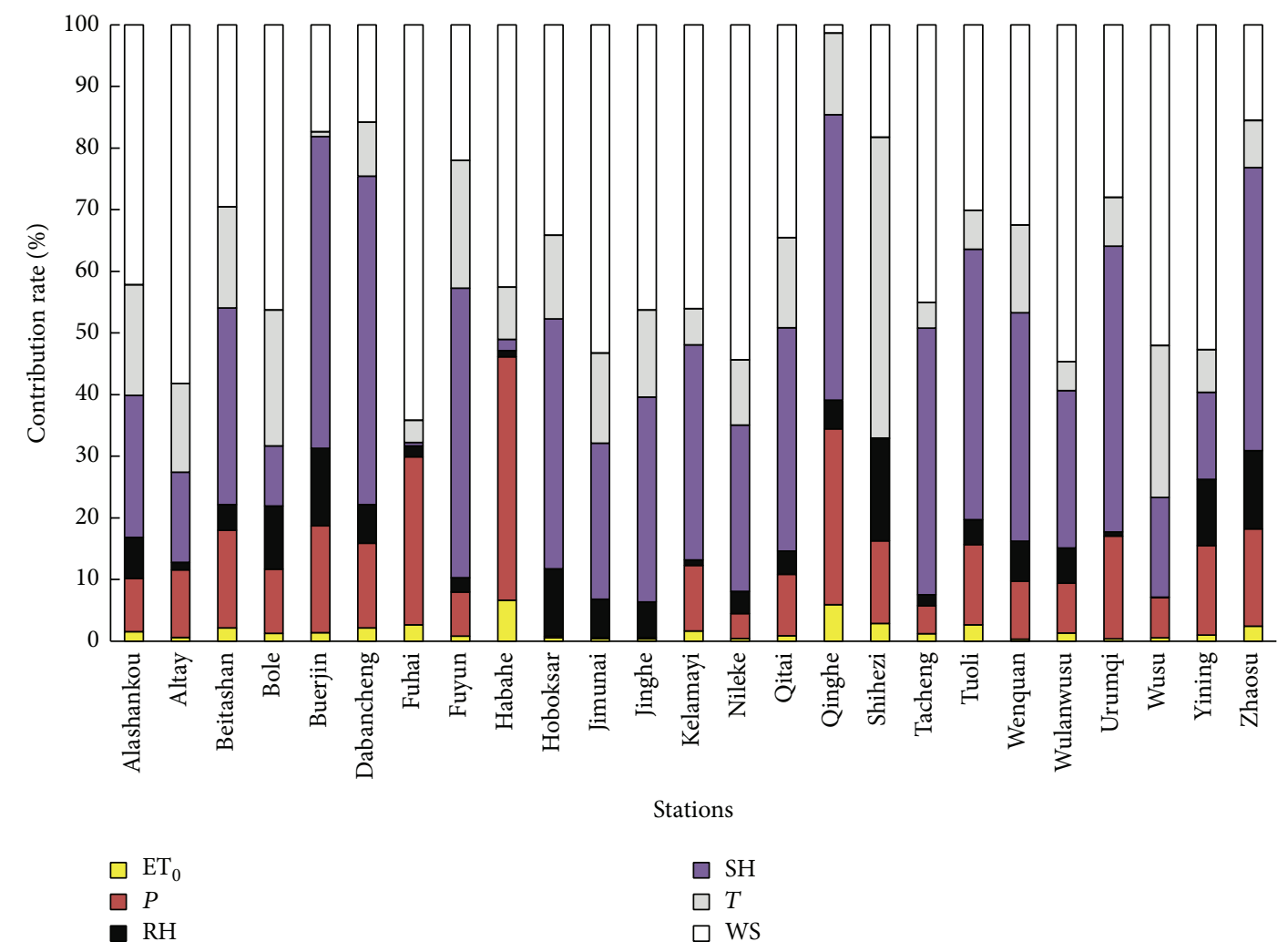

FIGURE 8: Percentage of contribution rate of meteorological factors to AI changes in North Xinjiang, China.

supply and local demand is also very large [51-53]. More efficient and effective water resource management strategies should be undertaken to adjust to the arid climate pattern and ever-growing water demand for local development in NX.

Aridity change not only is caused by variation of climate factors in NX, but also is the result of large-scale, persistent, atmospheric, and oceanic circulations patterns or regional geography and topography [54]. For example, the cause of aridity is significant within the context of global climate change, which may impact global atmospheric and oceanic circulation patterns. Furthermore, we only focus on annual averages and conduct no consideration on the seasonal change of aridity as well as climate factors. Therefore, other factors not considered here in the analysis are also important 


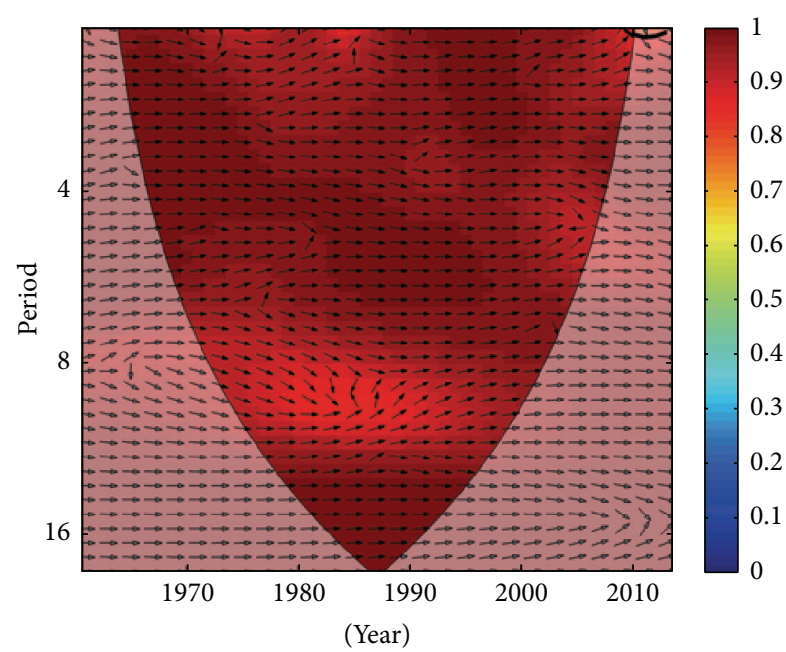

(a) $P$

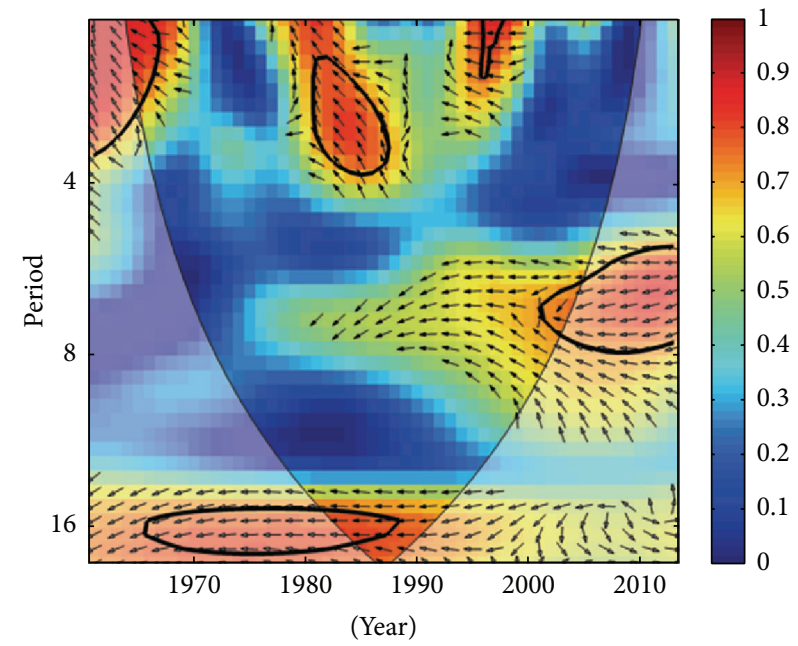

(c) $\mathrm{T}$

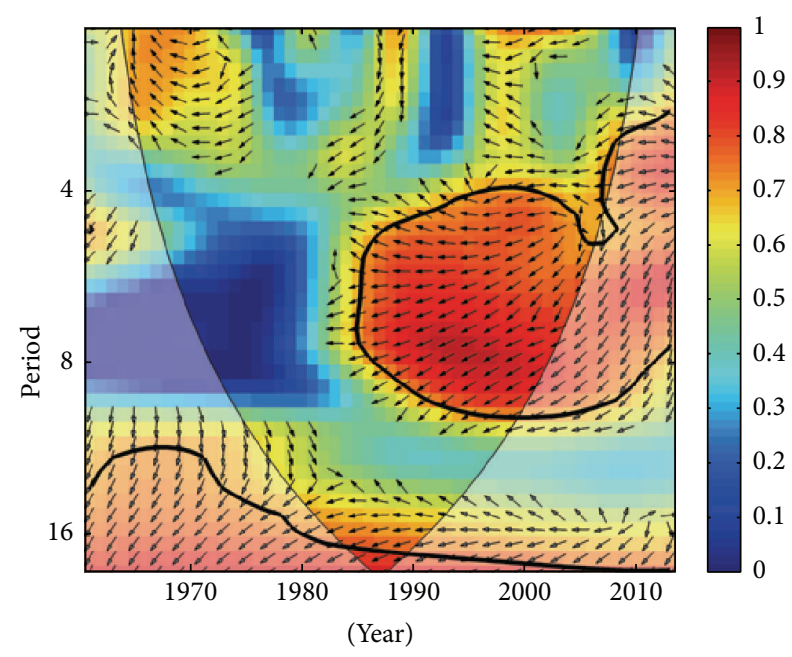

(e) $\mathrm{SH}$

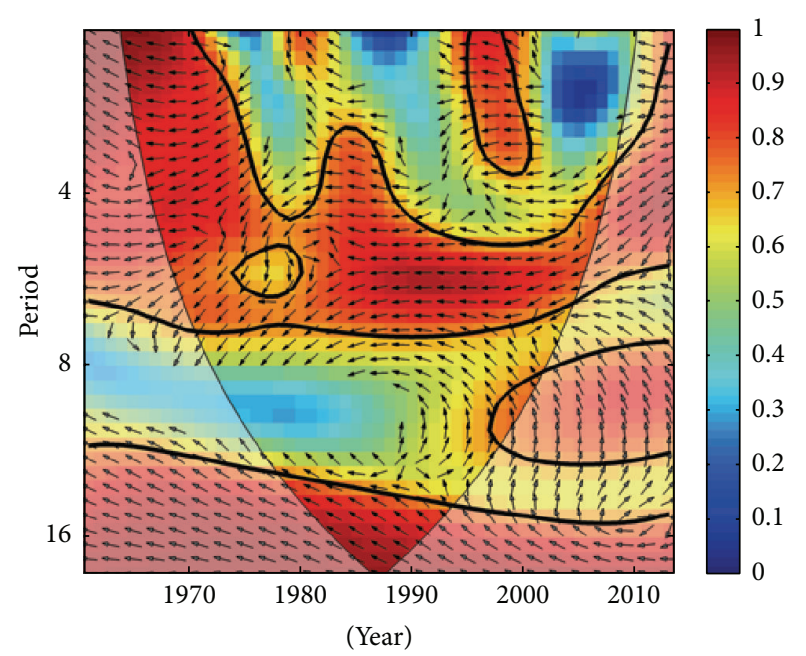

(b) $\mathrm{ET}_{0}$

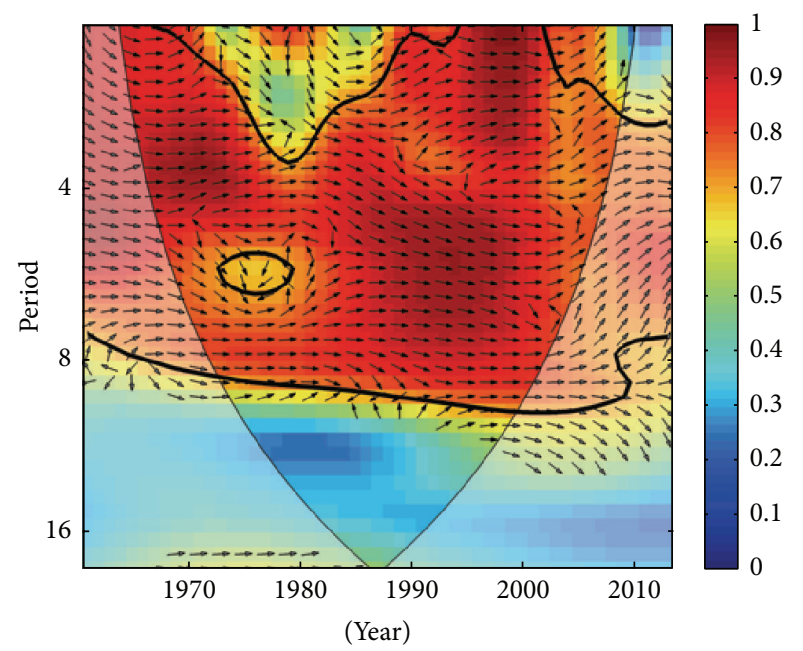

(d) $\mathrm{RH}$

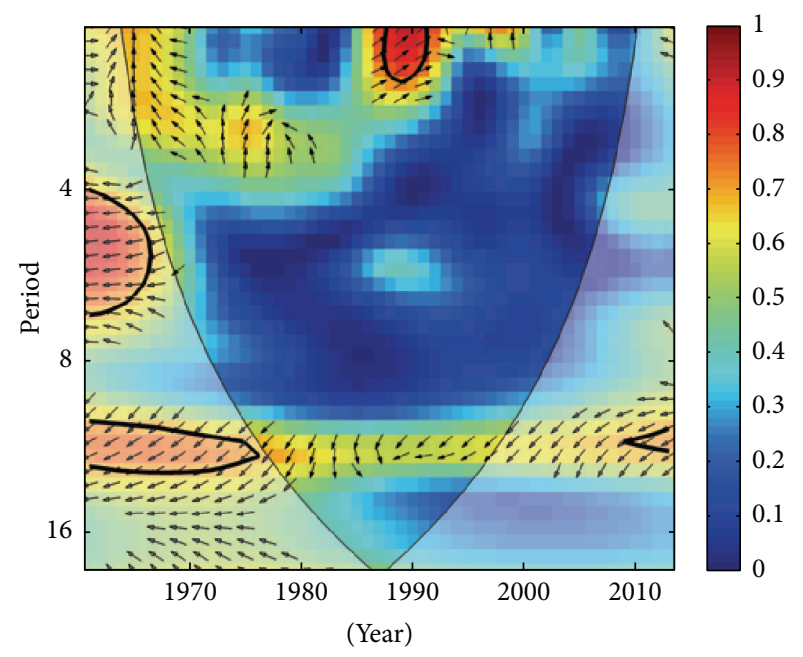

(f) WS

FIGURE 9: Wavelet coherence between annual AI and meteorological factors in North Xinjiang, China. The black contour lines show the regions of power significant at the 95\% level, which are computed based on 1000 Monte Carlo simulations. The cone of influence (black curve) indicates the region without edge effects. The power values are coded from dark blue for low power to dark red for high power, as shown in the right panel on each figure. The vectors indicate the phase difference between the aridity and annual meteorological factors: $\rightarrow$ : in phase; $\leftarrow$ : in antiphase; $\uparrow: X$ leading $Y$ by $90^{\circ}$; $\downarrow: Y$ leading $X$ by $90^{\circ}$. 
and can contribute to the influence of aridity. In addition, the aridity had a turning point in the late 1980s and the early 1990s. The sensitivity and contribution of AI to climatic factors may be different before and after the turning point, which deserves to carry out some further research on how meteorological factors impose influence on the variation of aridity in the dry period and the wet period, respectively.

\section{Conclusions}

This study assessed the long-term trend and spatial distribution of aridity index in North Xinjiang, China, an arid region highly sensitive to climate change. We found that the region is becoming more humid over the past 50 years and that the decline in sunshine hours and wind speed are the main causes for the increasing aridity index. This finding is encouraging for resource managers to develop adaptive strategies and plans for regional conservation and sustainability, although potential droughts and negative impacts should not be unexpected. Future studies should also pay attention to the local runoff variations, agricultural irrigation patterns, and vegetation variability.

\section{Competing Interests}

The authors declare no competing interests.

\section{Acknowledgments}

This research was supported by the CAS/SAFEA International Partnership Program for Creative Research Teams (Y4a1011001), the Scientific Innovation Research Project for Graduate Students of Xinjiang (XJGRI2014079), and Soil Science Key Discipline Project of Xinjiang Uygur Autonomous Region.

\section{References}

[1] M. S. Al-Kalbani, M. F. Price, A. Abahussain, M. Ahmed, and T. O'Higgins, "Vulnerability assessment of environmental and climate change impacts on water resources in $\mathrm{Al} \mathrm{Jabal} \mathrm{Al}$ Akhdar, Sultanate of Oman," Water, vol. 6, no. 10, pp. 3118-3135, 2014.

[2] V. Ramanathan, P. J. Crutzen, J. T. Kiehl, and D. Rosenfeld, "Atmosphere: aerosols, climate, and the hydrological cycle," Science, vol. 294, no. 5549, pp. 2119-2124, 2001.

[3] J. Scheff and D. M. W. Frierson, "Terrestrial aridity and its response to greenhouse warming across CMIP5 climate models," Journal of Climate, vol. 28, no. 14, pp. 5583-5600, 2015.

[4] M. A. Asadi Zarch, B. Sivakumar, and A. Sharma, "Assessment of global aridity change," Journal of Hydrology, vol. 520, pp. 300313, 2015.

[5] B. Jia, Z. Zhang, L. Ci, Y. Ren, B. Pan, and Z. Zhang, "Oasis land-use dynamics and its influence on the oasis environment in Xinjiang, China," Journal of Arid Environments, vol. 56, no. 1, pp. 11-26, 2004.

[6] F. Zhang, T. Tiyip, V. C. Johnson et al., "The influence of natural and human factors in the shrinking of the Ebinur Lake,
Xinjiang, China, during the 1972-2013 period," Environmental Monitoring and Assessment, vol. 187, no. 1, pp. 1-14, 2015.

[7] X. Li and X. Ye, "Spatiotemporal characteristics of dry-wet abrupt transition based on precipitation in Poyang Lake basin, China," Water, vol. 7, no. 5, pp. 1943-1958, 2015.

[8] P. T. Nastos, N. Politi, and J. Kapsomenakis, "Spatial and temporal variability of the Aridity Index in Greece," Atmospheric Research, vol. 119, pp. 140-152, 2013.

[9] J. F. Schyns, A. Y. Hoekstra, and M. J. Booij, "Review and classification of indicators of green water availability and scarcity," Hydrology and Earth System Sciences, vol. 19, no. 11, pp. 45814608, 2015.

[10] C. Agnew and E. Anderson, Water Resources in the Arid Realm, Routledge, 1992.

[11] M. I. Budyko, "The heat balance of the earth's surface," Soviet Geography, vol. 2, no. 4, pp. 3-13, 1961.

[12] A. C. Costa and A. Soares, "Local spatiotemporal dynamics of a simple aridity index in a region susceptible to desertification," Journal of Arid Environments, vol. 87, pp. 8-18, 2012.

[13] J. Zambakas, General Climatology, Department of Geology, National \& Kapodistrian University of Athens, Athens, Greece, 1992.

[14] E. De Martonne, "Une nouvelle fonction climatologique: l'indice d'aridité," La Météorologie, vol. 2, pp. 449-458, 1926.

[15] I. Muhire and F. Ahmed, "Spatiotemporal trends in mean temperatures and aridity index over Rwanda," Theoretical and Applied Climatology, vol. 123, no. 1-2, pp. 399-414, 2016.

[16] M. Türkeş, U. M. Sümer, and İ. Demir, "Re-evaluation of trends and changes in mean, maximum and minimum temperatures of Turkey for the period 1929-1999," International Journal of Climatology, vol. 22, no. 8, pp. 947-977, 2002.

[17] K.-X. Zhang, S.-M. Pan, W. Zhang et al., "Influence of climate change on reference evapotranspiration and aridity index and their temporal-spatial variations in the Yellow River Basin, China, from 1961 to 2012," Quaternary International, vol. 380381, pp. 75-82, 2015.

[18] UNESCO, "Map of the world distribution of arid regions," MAB Technical Notes 7, UNESCO, Paris, France, 1979.

[19] H. Tabari and M.-B. Aghajanloo, "Temporal pattern of aridity index in Iran with considering precipitation and evapotranspiration trends," International Journal of Climatology, vol. 33, no. 2, pp. 396-409, 2013.

[20] H. Tabari, P. H. Talaee, S. S. M. Nadoushani, P. Willems, and A. Marchetto, "A survey of temperature and precipitation based aridity indices in Iran," Quaternary International, vol. 345, pp. 158-166, 2014.

[21] L.-G. Hou, S.-B. Zou, H.-L. Xiao, and Y.-G. Yang, "Sensitivity of the reference evapotranspiration to key climatic variables during the growing season in the Ejina oasis northwest China," Springer Plus, vol. 2, no. 1, article S4, 2013.

[22] Z. Liu, P. Zhou, F. Zhang, X. Liu, and G. Chen, "Spatiotemporal characteristics of dryness/wetness conditions across Qinghai Province, Northwest China," Agricultural and Forest Meteorology, vol. 182-183, pp. 101-108, 2013.

[23] Y. F. Shi, Y. P. Shen, and R. J. Hu, "Preliminary Study on signal, impact and foreground of climatic shift from warm-dry to warm-humid in northwest China," Journal of Glaciology and Geocryology, vol. 24, no. 3, pp. 219-226, 2002 (Chinese).

[24] Q. Geng, P. Wu, Q. Zhang, X. Zhao, and Y. Wang, "Dry/wet climate zoning and delimitation of arid areas of Northwest China based on a data-driven fashion," Journal of Arid Land, vol. 6, no. 3, pp. 287-299, 2014. 
[25] Y. Li and M. Zhou, "Trends in dryness index based on potential evapotranspiration and precipitation over 1961-2099 in Xinjiang, China," Advances in Meteorology, vol. 2014, Article ID 548230, 15 pages, 2014.

[26] L. Wang, H. L. Liu, A. M. Bao, X. L. Pan, and X. Chen, "Estimating the sensitivity of runoff to climate change in an alpine-valley watershed of Xinjiang, China," Hydrological Sciences Journal, vol. 61, no. 6, pp. 1069-1079, 2016.

[27] X. Zhang, Y. Ren, Z.-Y. Yin, Z. Lin, and D. Zheng, "Spatial and temporal variation patterns of reference evapotranspiration across the Qinghai-Tibetan Plateau during 1971-2004," Journal of Geophysical Research Atmospheres, vol. 114, no. 15, pp. 44274433, 2009.

[28] F. Q. Jiang, C. Zhu, and R. J. Hu, "Trend analysis of precipitation over northern Xinjiang for the period 1961-1997," Scientia Geographica Sinica, vol. 22, no. 669-672, 2002.

[29] C. Xu, J. Li, J. Zhao, S. Gao, and Y. Chen, "Climate variations in northern Xinjiang of China over the past 50 years under global warming," Quaternary International, vol. 358, pp. 83-92, 2015.

[30] B. Zhu, J. Yu, P. Rioual, Y. Gao, Y. Zhang, and H. Xiong, "Climate effects on recharge and evolution of natural water resources in middle-latitude watersheds under arid climate," in Environmental Management of River Basin Ecosystems, pp. 91-109, Springer International Publishing, Cham, Switzerland, 2015.

[31] X. Y. Tang, X. L. Tang, and J. M. Zhang, "Arid climate change features in Xinjiang, China, during 1961-2011," Journal of Applied Sciences, vol. 14, no. 20, pp. 2570-2577, 2014.

[32] M. Wu, Y. Chen, H. Wang, and G. Sun, "Characteristics of meteorological disasters and their impacts on the agricultural ecosystems in the northwest of China: a case study in Xinjiang," Geoenvironmental Disasters, vol. 2, no. 1, pp. 1-10, 2015.

[33] R. G. Allen, L. S. Pereira, D. Raes, and M. Smith, Crop Evapotranspiration-Guidelines for Computing Crop Water Requirements-FAO Irrigation and Drainage Paper 56, FAO, Rome, Italy, 1998.

[34] K. Beven, "A sensitivity analysis of the Penman-Monteith actual evapotranspiration estimates," Journal of Hydrology, vol. 44, no. 3-4, pp. 169-190, 1979.

[35] Y. Gao, X. Li, L. R. Leung, D. Chen, and J. Xu, "Aridity changes in the Tibetan Plateau in a warming climate," Environmental Research Letters, vol. 10, no. 3, pp. 34013-34024, 2015.

[36] F. Hupet and M. Vanclooster, "Effect of the sampling frequency of meteorological variables on the estimation of the reference evapotranspiration," Journal of Hydrology, vol. 243, no. 3-4, pp. 192-204, 2001.

[37] H. X. Zheng and J. Liu, "Long-term trends of aridity index and its sensitivity to climate factors in Northeast China: 1971-2008," Journal of Geographical Research, vol. 20, no. 10, pp. 1765-1774, 2011 (Chinese).

[38] R. H. McCuen, "A sensitivity and error analysis cf procedures used for estimating evaporation," JAWRA Journal of the American Water Resources Association, vol. 10, no. 3, pp. 486-497, 1974.

[39] C. Xu, Y. Chen, Y. Yang, X. Hao, and Y. Shen, "Hydrology and water resources variation and its response to regional climate change in Xinjiang," Journal of Geographical Sciences, vol. 20, no. 4, pp. 599-612, 2010.

[40] T. Gunda, G. M. Hornberger, and J. M. Gilligan, "Spatiotemporal patterns of agricultural drought in Sri Lanka: 1881-2010," International Journal of Climatology, vol. 190, pp. 75-81, 2015.
[41] M. Lafrenière and M. Sharp, "Wavelet analysis of inter-annual variability in the runoff regimes of glacial and nival stream catchments, Bow Lake, Alberta," Hydrological Processes, vol. 17, no. 6, pp. 1093-1118, 2003.

[42] S. Jevrejeva, J. C. Moore, and A. Grinsted, "Influence of the Arctic Oscillation and El Nin-Southern Oscillation (ENSO) on ice conditions in the Baltic Sea: the wavelet approach," Journal of Geophysical Research Atmospheres, vol. 108, no. 21, pp. 10-11, 2003.

[43] N. A. Rayner, D. E. Parker, E. B. Horton et al., "Global analyses of sea surface temperature, sea ice, and night marine air temperature since the late nineteenth century," Journal of Geophysical Research D: Atmospheres, vol. 108, no. 14, pp. -1-29, 2003.

[44] A. Grinsted, J. C. Moore, and S. Jevrejeva, "Application of the cross wavelet transform and wavelet coherence to geophysical times series," Nonlinear Processes in Geophysics, vol. 11, no. 5-6, pp. 561-566, 2004.

[45] C. Torrence and P. J. Webster, "Interdecadal changes in the ENSO-monsoon system," Journal of Climate, vol. 12, no. 8, pp. 2679-2690, 1999.

[46] S. Piao, P. Ciais, Y. Huang et al., "The impacts of climate change on water resources and agriculture in China," in Regional Climate Studies of China, pp. 447-464, Springer, Berlin, Germany, 2008.

[47] S. Feng and Q. Fu, "Expansion of global drylands under a warming climate," Atmospheric Chemistry and Physics, vol. 13, no. 19, pp. 10081-10094, 2013.

[48] Y. Yin, S. Wu, G. Chen, and E. Dai, "Attribution analyses of potential evapotranspiration changes in China since the 1960s," Theoretical and Applied Climatology, vol. 101, no. 1, pp. 19-28, 2010.

[49] G. Gao, D. Chen, G. Ren, Y. Chen, and Y. Liao, "Spatial and temporal variations and controlling factors of potential evapotranspiration in China: 1956-2000," Journal of Geographical Sciences, vol. 16, no. 1, pp. 3-12, 2006.

[50] Y. H. Yin, S. H. Wu, and E. F. Dai, "Determining factors in potential evapotranspiration changes over China in the period 1971-2008," Chinese Science Bulletin, vol. 55, no. 29, pp. 33293337, 2010.

[51] Y. Bai, H. Xu, and H. Ling, "Drought-flood variation and its correlation with runoff in three headstreams of Tarim River, Xinjiang, China," Environmental Earth Sciences, vol. 71, no. 3, pp. 1297-1309, 2014.

[52] C. Li, X. Zhou, J. Sun, F. Li, Y. Gao, and H. Wang, "Evapotranspiration model of maize field with ridge culture under alternate furrow irrigation," Irrigation and Drainage, vol. 64, no. 4, pp. 557-565, 2015.

[53] C. Cassardo and J. A. A. Jones, "Managing water in a changing world," Water, vol. 3, no. 2, pp. 618-628, 2011.

[54] R. Maliva and T. Missimer, "Arid lands water evaluation and management," in Environmental Science and Engineering, Springer, Berlin, Germany, 2012. 

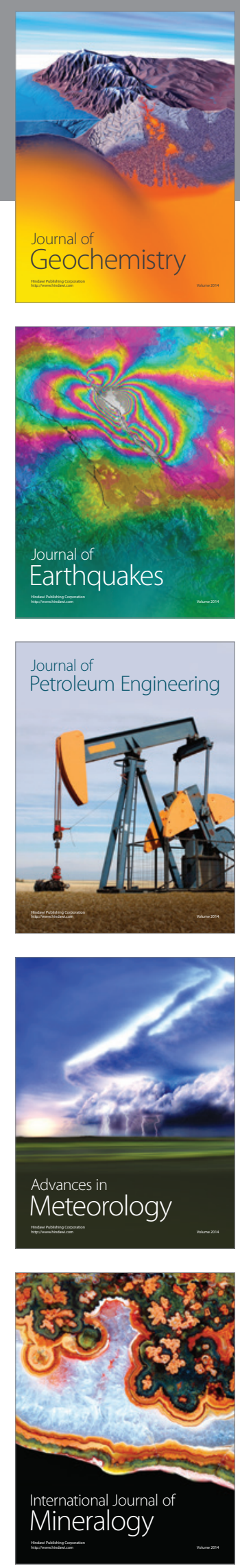
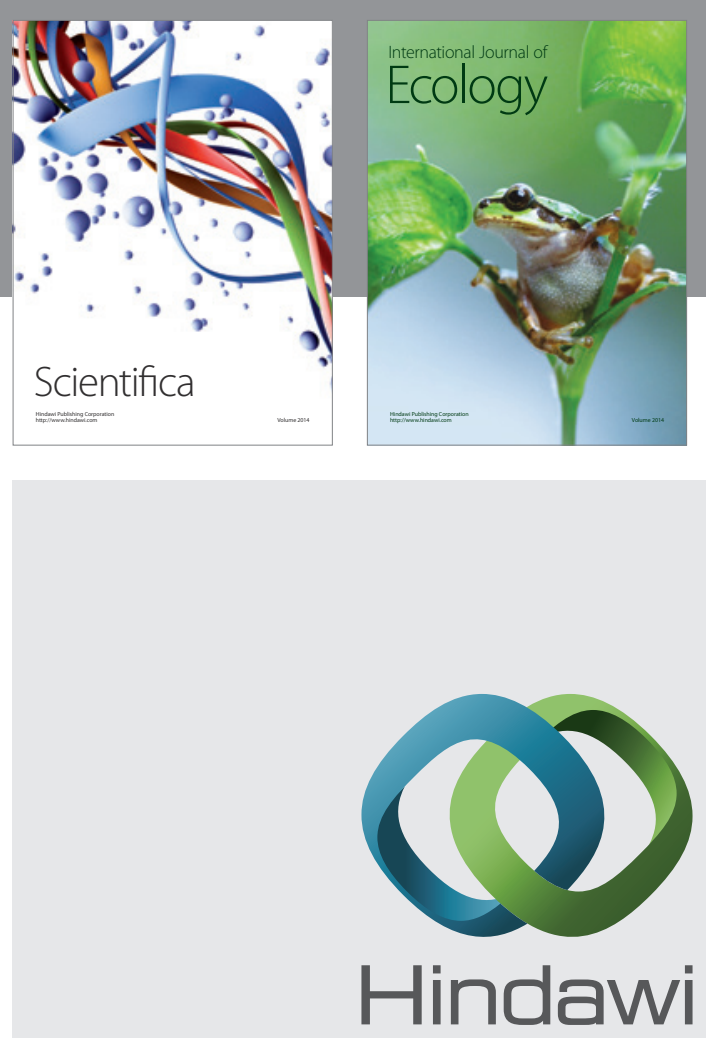

Submit your manuscripts at

http://www.hindawi.com
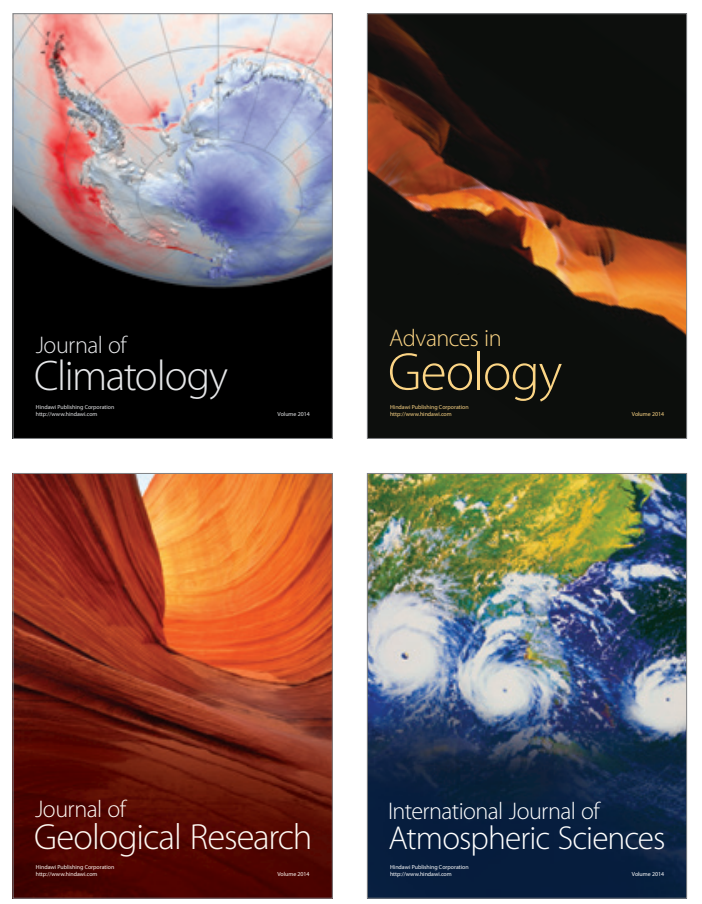

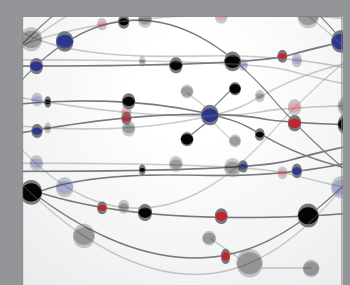

The Scientific

\section{World Journal}
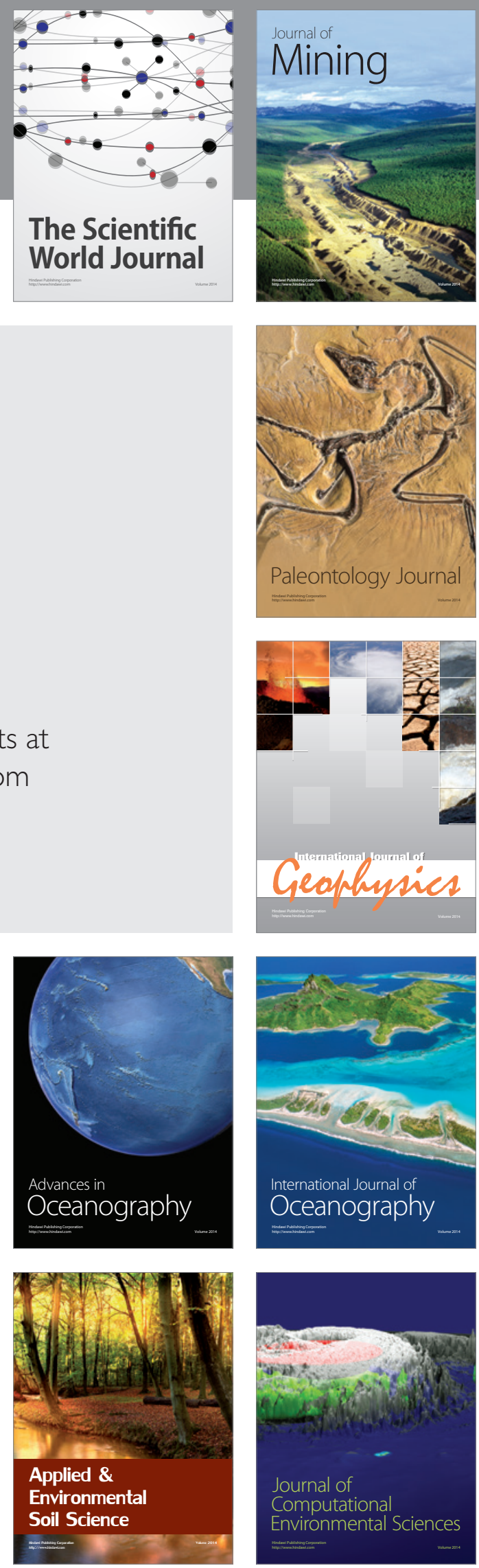